\title{
Protein Lysine Acetylated/Deacetylated Enzymes and the Metabolism-Related Diseases
}

\author{
Qilin Wang1* , Shangjing Guo², Yaman Gao \\ ${ }^{1}$ Liaocheng University School of Life Sciences, Liaocheng, China \\ ${ }^{2}$ Liaocheng University School of Pharmacy, Liaocheng, China \\ Email: *wql@lcu.edu.cn
}

How to cite this paper: Wang, Q.L., Guo, S.J. and Gao, Y.M. (2016) Protein Lysine Acetylated/Deacetylated Enzymes and the Metabolism-Related Diseases. Advances in Bioscience and Biotechnology, 7, 454-467. http://dx.doi.org/10.4236/abb.2016.711044

Received: September 5, 2016

Accepted: November 15, 2016

Published: November 18, 2016

Copyright $\odot 2016$ by authors and Scientific Research Publishing Inc. This work is licensed under the Creative Commons Attribution International License (CC BY 4.0).

http://creativecommons.org/licenses/by/4.0/

\section{(c) (i) Open Access}

\begin{abstract}
Lysine acetylation is a reversible posttranslational modifcation, an epigenetic phenomenon, referred to as transfer of an acetyl group from acetyl CoA to lysine $\varepsilon$ amino group of targeted protein, which is modulated by acetyltransferases (histone/ lysine (K) acetyltransferases, HATs/KATs) and deacetylases (histone/lysine (K) deacetylases, HDACs/KDACs). Lysine acetylation regulates various metabolic processes, such as fatty acid oxidation, Krebs cycle, oxidative phosphorylation, angiogenesis and so on. Thus disorders of lysine acetylation may be correlated with obesity, diabetes and cardiovascular disease, which are termed as the metabolic complication. With accumulating studies on proteomic acetylation, lysine acetylation also involves in cell immune status and degenerative diseases, for example, Alzheimer's disease and Huntington's disease. This review primarily summarizes the current studies of lysine acetylation in metabolism modulation and in metabolism-related diseases, such as cardiovascular disease and fat metabolism disorder.
\end{abstract}

\section{Keywords}

Lysine Acetylation, Acetyl CoA, Metabolism-Related Disease, Cardiovascular Disease, Obesity

\section{Introduction}

Lysine acetylation is a wide posttranslational modification of proteins, extending from the minority in nucleus to the majority in cytoplasm. In a specific cell, not less than 2000 lysine acetylated proteins are present. These acetylated proteins include metabolic enzymes, cytoskeletal proteins, molecular chaperones, ribosomal proteins, nuclear transport factors and so on, which accordingly participate in metabolism, cell signal transduction, stress reaction, protein hydrolysis, cellular apoptosis and growth of neurons, etc. Proteomic analysis on acetylation has showed that most acetylated proteins in 
cytoplasm and mitochondria are correlated with intermediary metabolism [1]. In the process of intermediary metabolism, lysine acetylation regulates metabolic enzymes by at least two mechanisms: 1) regulation of enzyme catalytic activity; 2) effect on the stability of enzyme [2] [3]. Metabolic disorders frequently happen in diseases, such as obesity, diabetes, cardiovascular disease and cancer, and therefore lysine acetylation may exert certain functions in these diseases' genesis [4] [5]. Recent accumulating studies have found that neurodegenerative diseases, such as Alzheimer's disease and Huntington's syndrome, are also correlated with protein lysine acetylation [6] [7]. Therefore, regulation of protein lysine acetylation may be an effective strategy for treatment of metabolism-related diseases.

Lysine acetylation is referred to as transfer of an acetyl group to lysine $\mathcal{\varepsilon}$-amino group of targeted protein, modulated by acetyltransferases and deacetylases in common. Deacetylases KDACs already found are divided into four categories: class I includes KDAC1, KDAC2, KDAC3 and KDAC8; class II includes KDAC4 KDAC10 (except KDAC8); class III includes Sirtuins 1 - 7; class IV includes KDAC11. The classes I, II, and IV KDACs share sequence homology (identity, 24\% - 65\%; similarity, 41\% - 82\%) on the deacetylase domain, and their activities depend on $\mathrm{Zn}^{2+}$ ion [8]. The class III Sirtuin deacetylases' activities are $\mathrm{NAD}^{+}$-dependent and response to the nutrition status and energy charge in vivo. Different KDACs and Sirtuins may locate in diverse cellular compartments (see Table 1), and participate in different gene expression regulation and acetylation modification of various functional proteins. Acetyltransferase HATs/KATs are primarily divided into three groups: 1) GCN5-related N-acetyltransferases (GNAT); 2) E1A-related proteins: P300/KAT3A and CBP (KAT3B); 3) MYST proteins [9]. The p300/CBP and GCN5 are the most characteristic acetyltransferase families with the strongest enzymatic activity. Extensively characterized acetyltransferases are predominantly known as nuclear enzymes, although they also function in cytoplasm under some circumstances. Furthermore, acetyltransferases now include nuclear receptor coactivators and others (see Table 2). These enzymes and proteins profoundly influence behavior and physiology of organisms, and so extensive studies on protein lysine acetylation are in progress [10]-[12]. Acetyltransferases and deacetylases determine the acetylated status in vivo by co-modulating acetylation/deacetylation of intracellular proteins, and may play important roles in regulation of intermediary metabolism and the metabolism-related diseases.

\section{Protein Lysine Acetylation and Intermediary Metabolism}

By proteomic acetylaiton analysis, more than $20 \%$ proteins and enzymes in eukaryotic hepatic mitochondria are acetylated, and most of acetylation modules response to acute malnutrition. Enzymes that are involved in transformation of carbon source, are mostly acetylated in Krebs cycle and beta oxidation in mitochondria [13]. Sirt3, as a member of Sirtuin family, directly regulates activities of mitochondrial acetyl CoA synthetase 2 (AceCS2) [14], long chain fatty acetyl CoA dehydrogenase (LCAD) [15], and isocitrate dehydrogenase (IDH2) by $\mathrm{NAD}^{+}$-dependent deacetylation [16]. Recently it has been 
Table 1. Lysine (K) deacetylases classification and localization.

\begin{tabular}{|c|c|c|c|}
\hline \multicolumn{3}{|c|}{ Name } & Localization \\
\hline \multicolumn{4}{|l|}{ Class I } \\
\hline \multicolumn{3}{|c|}{ KDAC1 } & Nucleus \\
\hline \multicolumn{3}{|c|}{ KDAC2 } & Nucleus \\
\hline \multicolumn{3}{|c|}{ KDAC3 } & Nucleus/cytoplasm \\
\hline \multicolumn{3}{|c|}{ KDAC8 } & Cytoplasm \\
\hline \multicolumn{4}{|l|}{ Class II a } \\
\hline \multicolumn{3}{|c|}{ KDAC4 } & Cytoplasm Nucleus \\
\hline \multicolumn{3}{|c|}{ KDAC5 } & Cytoplasm Nucleus \\
\hline \multicolumn{3}{|c|}{ KDAC7 } & Cytoplasm Mitochondria Nucleus \\
\hline \multicolumn{3}{|c|}{ KDAC9 } & Cytoplasm Nucleus \\
\hline \multicolumn{4}{|c|}{ Class II b } \\
\hline \multicolumn{3}{|c|}{ KDAC6 } & Cytoplasm \\
\hline \multicolumn{3}{|c|}{ KDAC10 } & Cytoplasm Nucleus \\
\hline \multirow[t]{8}{*}{ Class III } & (Sirtuins) & $(\mathrm{kDa})$ & \\
\hline & Sirt1 & 120 & Cytoplasm Nucleus \\
\hline & Sirt2 & 43 & Cytoplasm Nucleus \\
\hline & Sirt3 & $28 / 44(\mathrm{~L})$ & Mitochondria Nucleus \\
\hline & Sirt4 & 35 & Mitochondria \\
\hline & Sirt5 & 34 & Mitochondria \\
\hline & Sirt6 & 37 & Nucleus \\
\hline & Sirt7 & 45 & Nucleus \\
\hline \multicolumn{4}{|l|}{ Class IV } \\
\hline \multicolumn{3}{|c|}{ KDAC11 } & Nucleus \\
\hline
\end{tabular}

found that Sirt5, another deacetylase in mitochondrial as Sirt3, also regulates LCAD [17]. As mentioned above, these key enzymes in energy metabolism and Krebs cycle may supply with necessary NADPH for mitochondrial antioxidant defense. Sirt3 also activates succinate dehydrogenase complex II, aconitase and as a result promotes oxidative phosphorylation [18]-[20]. Deacetylation of cyclophilin D by Sirt3 induces the interaction between hexokinase II and mitochondria more unsteady, beneficial to stimulate oxidative phosphorylation for metabolism [21]. From above results, Sirt3 may serve as an important modulating player in 
Table 2. Lysine (k) acetyltransferases classification and the former names in human.

\begin{tabular}{|c|c|}
\hline New name & Former name (human) \\
\hline \multicolumn{2}{|c|}{ GNAT family } \\
\hline KAT1 & HAT1 \\
\hline \multicolumn{2}{|l|}{ KAT2 } \\
\hline KAT2A & hGCN5 \\
\hline KAT2B & PCAF \\
\hline KAT9 & ELP3 \\
\hline \multicolumn{2}{|c|}{ P300/CBP family } \\
\hline \multicolumn{2}{|l|}{ KAT3 } \\
\hline KAT3A & $\mathrm{CBP}$ \\
\hline КАТ3В & P300 \\
\hline \multicolumn{2}{|c|}{ MYST family } \\
\hline KAT5 & TIP60 \\
\hline \multicolumn{2}{|l|}{ KAT6 } \\
\hline KAT6A & MOZ/MYST3 \\
\hline KAT6B & MORF/MYST4 \\
\hline KAT7 & HBO1/MYST2 \\
\hline KAT8 & hMOF/MYST1 \\
\hline \multicolumn{2}{|c|}{ Nuclear receptor coactivators } \\
\hline KAT13A & SRC-1 \\
\hline \multirow[t]{5}{*}{ KAT13B } & SRC-3 \\
\hline & TIF-2 \\
\hline & ATF-2 \\
\hline & GRIP1 \\
\hline & ACTR \\
\hline \multicolumn{2}{|l|}{ Others } \\
\hline KAT4 & TAF1 \\
\hline \multicolumn{2}{|l|}{ KAT10 } \\
\hline \multicolumn{2}{|l|}{ KAT11 } \\
\hline \multicolumn{2}{|l|}{ KAT12 } \\
\hline KAT13C & $\mathrm{P} 160$ \\
\hline KAT13D & CLOCK \\
\hline
\end{tabular}


fatty acid oxidation, Krebs cycle and oxidative phosphorylation.

In liver cells, deacetylase Sirt1 inhibits glycolysis and enhances gene expession of liver glyconeogenesis by deacetylating of lysine in peroxisome proliferator-activated receptor gamma coactivator 1-alpha (PGC-1 $\alpha$ ) and thus activating its enzyme activity. In muscles, Sirt1 may also promote mitochondrial function, energy balance, and oxygen consumption to induce oxidative phosphorylation and biogenesis within mitochondria [22]. In process of fatty acid metabolism, Sirt1 deacetylates and activates acetyl CoA synthetase 1 (AceCS1), a substrate of Sirt1, to enhance acetate into fatty acid metabolism [23]. Furthermore, Sirt6-knockout mice showed hypoglycemia, subcutaneous fat reduced, suggesting that Sirt6 may participate in maintaining the balance of blood sugar and fat synthesis in vivo [24]. In general, deacetylase Sirtuins play important regulatory roles in intermediary metabolism, and may intervene metabolic disorder by modulating the activities of intermediary enzymes in mammalian.

\section{Protein Lysine Acetylation and Metabolism-Related Diseases}

Lysine acetylation, as a primary posttranslational modification of enzymes in intermediary metabolism, is widely studied in mice/human liver cells, and also in human leukemia cells. Stdudies have showed that protein lysine acetylation invloves in metabolism of carbohydrates, lipids, amino acids, nucleotides, and the secondary metabolites, etc. Consequently, lysine acetylation modification is also associated with obesity, diabetes, cardiovascular disease and so on [25]. That is to say, lysine acetylation may be a primary regulatory mechanism for metabolism-related diseases, and the enzymes invloved in lysine acetylation/deacetylation may have intimate correlation with metabolism-related diseases.

\subsection{Protein Lysine Acetylation and Fat Metabolism Disorders}

Dietary obesity (DR) is correlated with type II diabetes and intra-adipose tissue hypoxia and activation of HIF- $1 \alpha$. Recent studies have found that Sirt2 is involved in metabolic diseases by modulation of HIF-1 $\alpha$ [26]. Importantly, in visceral adipose tissue from human obese subjects, the expression level of Sirt2 is very low while HIF-1 $\alpha$ is high. Depletion of HIF-1a in 3T3-L1 adipocytes causes induction of Sirt2 mRNA and protein, which indicates that Sirt2 dysfunction perhaps is the factor of obesity development. Thus, by negatively regulating the Sirt2-HIF-1 $\alpha$ regulatory axis may represent an effective preventation method in dietary obesity [27]. In another study, overexpression or reduction of cytoplasmic Sirt2 blocks or promotes adipogenesis respectively [28]. Many genes related with adipocyte differentiation, such as GLUT4, aP2 and fatty acid synthase genes, are all regulated by Sirt2, and thus regulate adipocyte differentiation. This is attributed to a direct interaction between Sirt 2 and acetylation patterns in controlling lipogenesis [29].

Deacetylase Sirt1 also promotes fat mobilization by inhibiting peroxisome proliferator activated receptor gamma (PPAR gamma) in adipocytes [30]. Over-expression of Sirt1 or inhibition of Sirt1 by siRNA weakened or promoted lipogenesis in 3T3-L1 cells 
respectively. In process of adipocyte differentiation, Sirt1 upregulation may promote lipolysis and fat decrease. Expressions of Sirt1 protein and mRNA accompanied with expression of C/EBP alpha, which regulates the expression of Sirt1 by binding on the Sirt1 promotor [31] [32]. Sirt1 also regulates the expression of adiponectin gene through the FoxO1-C/EBP alpha transcription complex [33]. The regulatory mechanism of Sirt1 and Sirt2 on lipogenesis is displayed in Figure 1.

Adipose tissue provides the reversible energy reserves for the body in form of fat. Adipocytes store excess fat by regulating their proliferous hypertrophy and hyperplasia in obesity [34] [35]. Adipocytes are the active components of adipose tissue, which are indirectly interacted by immune cells, such as macrophages, mononuclear cells, $\mathrm{T}$ cells and giant cells. Adipocytes and immune cells secrete different paracrine factors and endocrine factors (collectively referred to as adipocytokines) for normal physiological signal transduction. When excessive energy store as fat, the biological functions of adipocytes change [35]. That is to say, adipocytokines are secreted in a pathological state, and will recruit new adipocytes and immune cells to lead to metabolic dysfunction of adipocytes. Through broad-spectrum mass spectrometry and chemical analysis, when the activities of transcription factor C/EBP (CCAAT/enhancer binding protein), PPAR gamma (peroxisome proliferator-activated receptor gamma) and SREBP (sterol regulatory element-binding protein) were inhibited by KDAC isomer inhibitors in 3T3-L1 adipocytes in mice or in human preadipocytes, lipogenesis was blocked [36]. It has been demonstated by further study that the lipogenesis and differentiation of adipocytes depend on different KDAC isomer. KDAC1 and KDAC2 control lipogenesis, and have the positive regulatory effect on lipogenesis.

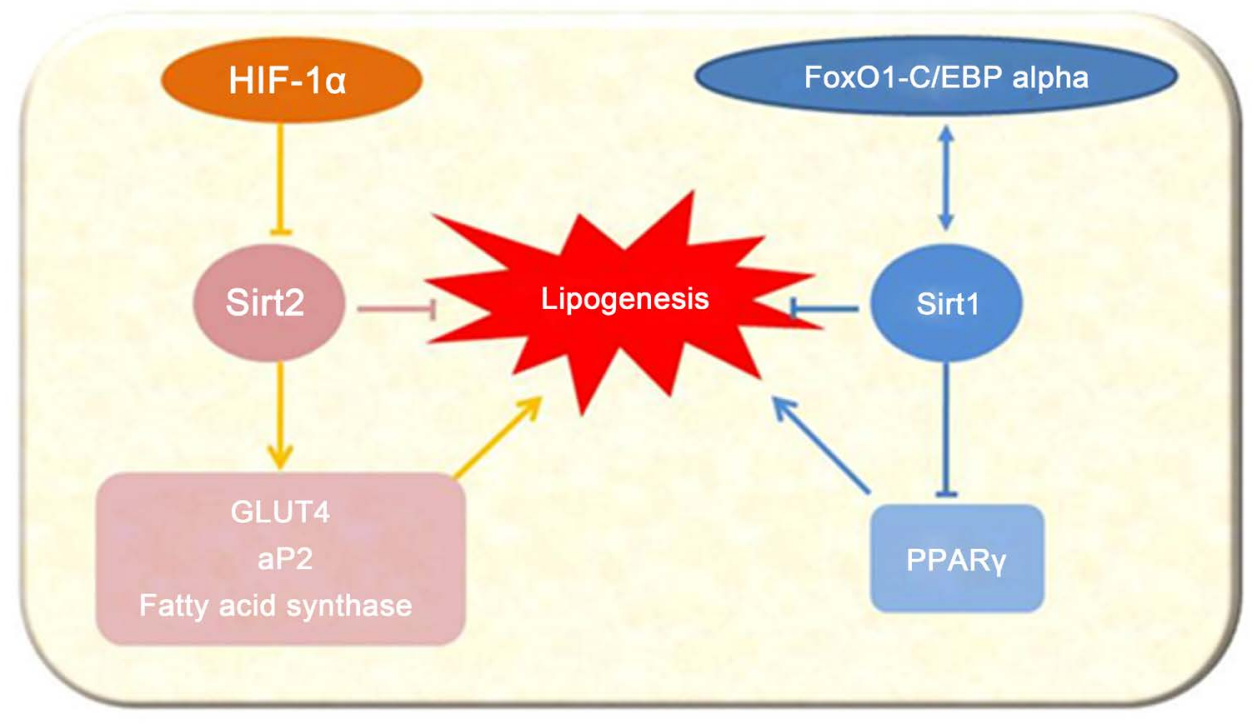

Figure 1. Deacetylase Sirt1 and Sirt2 block lipogenesis through different pathways. In 3T3-L1 cells the high expression of HIF-1 $\alpha$ inhibits Sirt2 expression and induces lipogenesis. While over expression of Sirt2 promotes adipocyte differentiation and inhibit lipogenesis by activation of GLUT4, aP2 and fatty acid synthase genes. As well, the interaction between Sirt1 and C/EBP alpha promotes the expression of Sirt1, which blocks lipogenesis by inhibiting PPAR $\gamma$. 
During cell differentiation in 3T3-L1 cells, gene promoter region of lipogenesis is highly selective acetylated, accompanied by the reduced expressions of KDAC1, KDA $\mathrm{C} 2$ and KDAC5, and the activities of global KDAC enzymes decrease [37]. In cultured mesenchymal progenitor cells, when KDAC1 and/or KDAC2 were/was knocked out, adipose accumulation reduced. KDAC9 plays the key negative regulatory roles for control adipose differentiation. For example, the adipose differentiation was inhibited in over-expressed KDAC9 precursor cells, while the adipose differentiation was increased in KDAC9-depleted precursor cells in mice by siRNA [38]. The experimental results above show that deacetylases not only regulate the transcription factors of lipogenesis, and their expression and activities also have key roles in regulating adipocyte differentiation. Dysfunction of deacetylases in vivo may lead to fat metabolism disorders and the related diseases.

\subsection{Protein Lysine Acetylation and Cardiovascular Disease}

Myocardial hypertrophy is an adaptive response for heart to maintain its function in condition of continuous load. Studies have shown that the size and function of heart are related with angiogenesis. When the cardiac muscle growth and angiogenesis were disturbed, adaptive myocardial hypertrophy in heart changed into heart disease [39]. Sirt1 is known expressed higher in vasculature of blood vessel. However, if Sirt1 was knocked down by siRNA in human umbilical vein endothelial cells, the gene expression related to vascular differentiation reduced, even the start of angiogenesis was supressed [40]. Another example was that in endothelial cells of mice lower limbs, the formation of cardiovascular induced by ischemia was inhibited when Sirt1 was silenced. Instead, the genes of vascular endothelial growth factor (VEFG) and its receptor 2 (VEFGR2) and nitric oxide synthase were expressed while resveratrol activated Sirt1 [41]. According to above experiments, Sirt1 induces angiogenesis probably by inhibiting forkhead box class $\mathrm{O}$ family transcription factor (FoxO1), an essential negative regulatory factor in vascular differentiation. Sirt1 interacts with FoxO1 and inactivates FoxO1 by deacetylation in human umbilical vein endothelial cells. In addition, another mechanism of angiogenesis may be that angiogenic factor is up-regulated directly, and simutaneously p53 (anti-angiogenesis factors) is inhibited. p53 expression has been studied up-regulated during the continuous pressure load on left ventricular, which in turn inhibits the activity of hypoxia inducing factor 1 (HIF-1), a transcription factor for gene expression under anoxic conditions. So inactivation of HIF-1 is easily understood associated with down-regulation of angiogenic factor expression, the reduced myocardial capillary density and the decrease of adaptive myocardial hypertrophy into heart disease. In myocardial cells, Sirt1 deacetylates p53 and subsequently reduce its activity [39], but HIF-1/2 induces the expression of Sirt1 protein [39] [42] [43]. Therefore, fine-tuning the balance between the activity of Sirt1 and p53 may decide the degree of angiogenesis in overload heart and the compensatory myocardial hypertrophy converted to the uncompensatory heart disease (see Figure 2).

In renin-angiotensin system, the signal protein angiotensin (angiotensin II) is often 


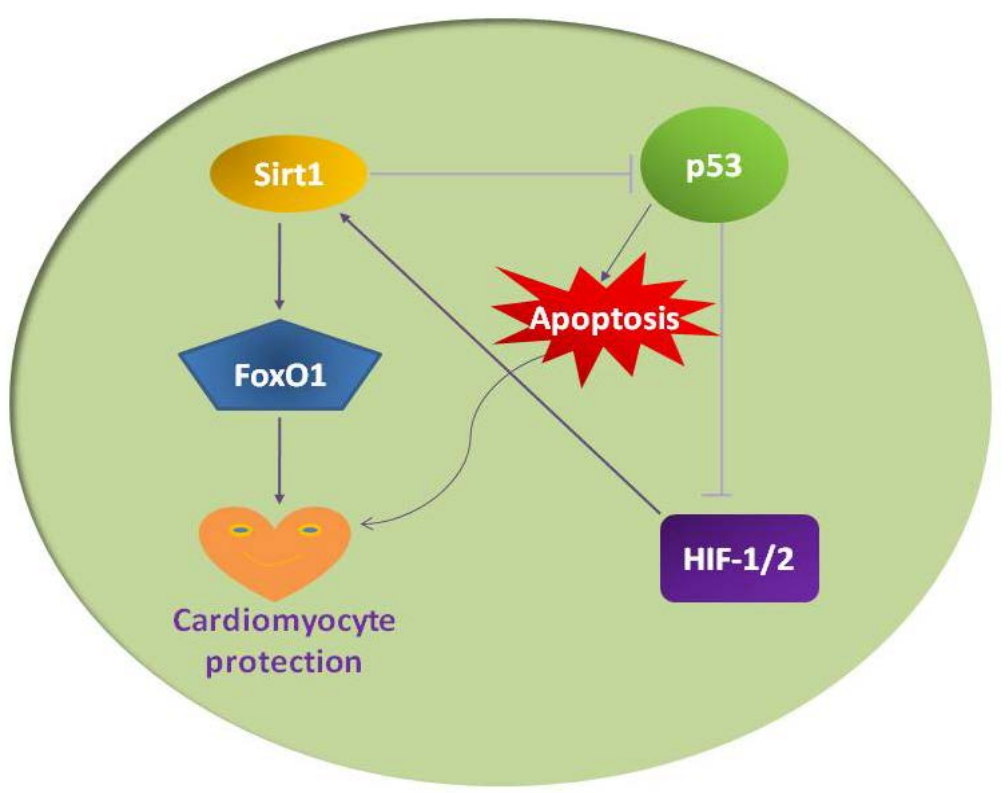

Figure 2. The regulatory mechanism between Sirt1 and p53 on cardiovascular protection. Sirt1 inhibits p53 activity by deacetylation. During the continuous pressure load on left ventricular, p53 inhibits the activity of HIF-1, while HIF-1/2 induces the expression of Sirt1. Sirt1 promotes cardiomyocyte protection by deacetylating FoxO1. On the other hand, p53 induces cellular apoptosis and also plays cardiomyocyte protection.

used to induce hypertension and myocardial hypertrophy in experimental animal models [44]. The heart attack in human or in pathological model is formed because of the change of proteins in renin-angiotensin system. If the receptor I of angiotensin II in mice was removed, the myocardial injury was reduced, and gene expresssions of Sirt3 and nicotinamide phosphoribosyltransferases (Nampt) were up-regulated [45]. Furthermore, exogenous $\mathrm{NAD}^{+}$addition to the hearts of mice may keep the levels of intracellular $\mathrm{NAD}^{+}$and suppress the myocardial hypertrophy induced by dope [46]. As well, in Sirt3 genetically modified mice, the expression of Nampt enzyme was over-expressed in a $\mathrm{NAD}^{+}$-dependent manner, and the myocardial infarction caused by myocardial ischemia reperfusion injury was reduced, all which suggested that the myocardial protective effect of $\mathrm{NAD}^{+}$is mediated by mitochondrial Sirt3 [46]. Besides, Sirt3 promotes deacetylation of dehydrogenase LCAD, respiratory chain complex I and cyclophilin $\mathrm{D}$, and stimulates the oxidative metabolism. It is generally believed that many cardiovascular diseases are associated with impaired energy supply, that is to say, the cardiomyocyte ATP and creatine pools in mitochondria have been depleted. It is consistent with study that in Sirt3-deficient mice, myocardial ATP decreased substantially in liver mitochondria and brown adipose tissue accompanied with hyperacetylation of the global mitochondrial proteins [47]. These data suggest that cardiovascular disease is caused by Sirt3 dysfunction in pathological state. In conclusion, mitochondrial Sirt 3 is a major deacetylase with heart protection, and may play a favorable role in treatment of cardiovascular disease. 


\section{The Possibility of Targeting Protein Lysine Acetylation}

During lysine acetylation process, proteins with bromodomain structure are recruited, and recently it has been found that proteins with AF9 YEATS domain have links with lysine acetylation [48]. As a result, catalytic enzymes for lysine acetylation are used as molecular targets of intervention. On the other hand, the proteins interacted with acetylated lysine also may be as potential molecular targets. Anacardic acid and garcinol are two natural products, and they are reported to inhibit acetyltransferase KAT3A/3B and KAT2B at the concentration of $5-10 \mu \mathrm{mol}$. Later studies have found that garcinol also interfer in KAT5. Curcumin selectively inhibits KAT3A/3B, rather than KAT2B [49]. Thiazole ketone, screened through high-throughput experiment, is the inhibitor of acetyltransferase KAT2B and KAT3A/3B [50]. Recently it has been developed several KATs inhibitors, such as natural product derivatives, small molecules, bi-substrate inhibitors and so on. These inhibitors may play a part in treatment of diseases ranging from cancer and inflammatory diseases to neurological disorders [51]. So these above compounds may have wide applications in clinic as the preferred agents in development of new antitumor drugs or anti-inflammatory drugs. Nevertheless, with the progressing of our knowledge on acetyltransferases KATs, targeting these enzymes may become possible. At the same time, it must be instructive to consider targeting acetyltransferase KATs comprehensively because KATs have various cellular substrates, ranging from histones and transcription factors to enzymes and nuclear receptors. Targeting these enzymes by inhibitors also consider the functions, the enzymatic activities and the substrate specificities.

In addition, some small molecules influence the transcriptional regulation of cancer cells and other cell processes by inhibition of combination of acetylated lysine in the hydrophobic pocket of Bromodomain [52]. Therefore, some small regulatory factors of KDACs have become the potential agents for treatment of cancer, heart disease and diabetes. For example, inhibitors of KDAC I and KDAC II have been designed and applied to the clinic as anticancer agents [53]. In our studies, the inhibitor of Sirt1/Sirt2 displays effect on cellular apoptosis in lung cancer. Studies have shown that these inhibitors may have wide functions in treatment of diseases such as inflammatory diseases, cardiovascular disease and so on [54] [55]. Furthermore, activators of KDAC class III (Sirtuins) as anti-aging and anti-cancer agents, have potential value for treatment of cardiovascular diseases and metabolic diseases [56]. It is worth considering that the functions of activators of KDAC class III are similar to the physiological effect of calorie restriction, suggesting KDAC class III (Sirtuins) are involved in energy storage and metabolism. Therefore, the studies of lysine acetylation and the corresponding molecular events after acetylation, may open up new avenues for identification of non- histone targets of KATs or KDACs, and for illustrating the molecular mechanism of lysine acetylation on chromosomal histones, and for improving the effectiveness of related drugs.

\section{Conclusion}

As discussed above, protein lysine acetylation plays important roles in metabolism-rela- 
ted diseases, such as cardiovascular disease and fat metabolism disorders. However, it should be considering that acetylated status is a comprehensive result by acetyltransferases and deacetylases in a specific cell and even in organism, perhaps correlated with other epigenetic modifications, such as methylation, phosphorylation and so on. If we would like to conclude with predicting that lysine acetylation is associated with certain disease, the acetylated status of proteins in vivo should be detected through proteomic survey approaches and high-throughput mass spectrometry-based proteomics. In this way, it may lead us to new candidates for this reversible modification and more new acetylated proteins, and even more new regulatory mechanism for metabolism, which may be benefical to intervention and treatment for diseases related with lysine acetylation.

\section{Perspective}

With the accumulating data in proteomics about lysine acetylation modification, more regulatory functions of lysine acetylation will be found, and the biological functions of acetylation might not limit to histones, cytoplasmic proteins and enzymes. Now, a major problem is whether there are links among the concentration of intracellular acetyl CoA, immune status of cells and chromatin, acetylated modes of metabolic enzymes. We hope to establish a model to determine that accumulation of intracellular acetyl CoA is subject to change following the change of cellular metabolism status, and to bypass different metabolic pathways, whether it simutaneously acts as a general control switch for acetylation status, the activities of metabolic enzymes and cellular immune status. By mass spectrometry analysis, inhibitors of KDACs or KATs induce different acetylated modes in different cell lines. So it should be to determine the effect of particular KDAC or KATs isomer on cell acetylation modes via massive protein combination experiments and single knockout experiments of KDAC or KATs isomers, and it should be considered these questions in design and development of new KDAC or KATs inhibitors or activators. According to the functional characteristics of KDAC or KATs inhibitors, drug design targeting specific tissues and organs may be even more beneficial, and reduce the adverse effect of miss target. Beyond that, it is very important to design and develop new, lower toxicity and selective KDAC or KATs regulatory molecules (inhibitors or activators), and they may be contributed in clinic for treatment of metabolism-related diseases, such as obesity, diabetes, cardiovascular disease and so on.

\section{Acknowledgements}

This work was supported by the grant from doctoral scientific research fund (No. 318051).

\section{References}

[1] Choudhary, C., Kumar, C., Gnad, F., et al. (2009) Lysine Acetylation Targets Protein Complexes and Co-Regulates Major Cellular Functions. Science, 325, 834-840. http://dx.doi.org/10.1126/science.1175371 
[2] Zhao, S.M., Xu, W., Jiang, W.Q., et al. (2010) Regulation of Cellular Metabolism by Protein Lysine Acetylation. Science, 327, 1000-1004. http://dx.doi.org/10.1126/science.1179689

[3] Wang, Q.J., Zhang, Y.K., Yang, C., et al. (2010) Acetylation of Metabolic Enzymes Coordinates Carbon Source Utilization and Metabolic Flux. Science, 327, 1004-1007.

http://dx.doi.org/10.1126/science.1179687

[4] Panchal, S.K. and Brown, L. (2011) Rodent Models for Metabolic Syndrome Research. Journal of Biomedicine and Biotechnology, 2011, Article ID: 351982. http://dx.doi.org/10.1155/2011/351982

[5] Zhang, K.L., Schrag, M., Crofton, A., Trivedi, R., Vinters, H. and Kirsch, W. (2012) Targeted Proteomics for Quantification of Histone Acetylation in Alzheimer's Disease. Protemics, 12, 1261-1268. http://dx.doi.org/10.1002/pmic.201200010

[6] Irwin, D.J., Cohen, T.J., Grossman, M., et al. (2012) Acetylated Tau, a Novel Pathological Signature in Alzheimer's Disease and Other Tauopathy. Brain, 135, 807-818. http://dx.doi.org/10.1093/brain/aws013

[7] Cohen, T.J., Friedmann, D., Hwang, A.W., Marmorstein, R. and Lee, V.M.Y. (2013) The Microtubule-Associated Tau Protein Has Intrinsic Acetyltransferase Activity. Nature Structural \& Molecular Biology, 20, 756-762. http://dx.doi.org/10.1038/nsmb.2555

[8] Yang, X.-J. and Seto, E. (2008) The Rpd3/Hda1 Family of Lysine Deacetylases: From Bacteria and Yeast to Mice and Men. Nature Reviews Molecular Cell Biology, 9, 206-218. http://dx.doi.org/10.1038/nrm2346

[9] Kjaergaard, M., Nørholm, A.B., Hendus-Altenburger, R., Pedersen, S.F., Poulsen, F.M. and Kragelund, B.B. (2010) Temperature-Dependent Structural Changes in Intrinsically Disordered Proteins: Formation of $\alpha$-Helices or Loss of Polyproline II? Protein Science, 19, 1555-1564. http://dx.doi.org/10.1002/pro.435

[10] Lonard, D.M. and O'Malley, B.W. (2013) Nuclear Receptor Coregulators: Modulators of Pathology and Therapeutic Targets. Nature Reviews Endocrinology, 8, 598-604. http://dx.doi.org/10.1038/nrendo.2012.100

[11] Wang, S.W., Jiang, B.W., Zhang, T.F., et al. (2015) Insulin and mTOR Pathway Regulate HDAC3-Mediated Deacetylation and Activation of PGK1. PLoS Biology, 13. e1002243. http://dx.doi.org/10.1371/journal.pbio.1002243

[12] Gainey, M.A., Tatavarty, V., Nahmani, M., Lin, H. and Turrigiano, G.G. (2015) Activity-Dependent Synaptic GRIP1 Accumulation Drives Synaptic Scaling up in Response to Action Potential Blockade. Proceedings of the National Academy of Sciences of the United States of America, 112, E3590-E3599. http://dx.doi.org/10.1073/pnas.1510754112

[13] Wellen, K.E., Hatzivassiliou, G., Sachdeva, U.M., Bui, T.V., Cross, J.R. and Thompson, C.B. (2009) ATP-Citrate Lyase Links Cellular Metabolism to Histone Acetylation. Science, 324, 1076-1080. http://dx.doi.org/10.1126/science.1164097

[14] Schwer, B., Bunkernborg, J., Verdin, R.O., Andersen, J.S. and Verdin, E. (2006) Reversible Lysine Acetylation Controls the Activity of the Mitochondrial Enzyme Acetyl-COA Synthetase 2. Proceedings of the National Academy of Sciences of the United States of America, 1003, 10224-10229. http://dx.doi.org/10.1073/pnas.0603968103

[15] Bharathi, S.S., Zhang, Y., Mohsen, A.W., et al. (2013) SIRT3 Regulates Long-Chain Acetyl-CoA Dehydrogenase by Deacetylating Conserved Lysines near the Active Site. The Journal of Biological Chemistry, 288, 33837-33847. http://dx.doi.org/10.1074/jbc.M113.510354

[16] Someya, S., Yu, W., Hallows, W.C., et al. (2010) Sirts Mediates Reduction of Oxidative Damage and Prevention of Age-Related Hearing Loss under Caloric Restriction. Cell, 143, 
802-812. http://dx.doi.org/10.1016/j.cell.2010.10.002

[17] Zhang, Y., Bharathi, S.S., Rardin, M.J., et al. (2015) SIRT3 and SIRT5 Regulates the Enzyme Activity and Cardiolipin Binding of Very Long-Chain Acyl-CoA Dehydrogenase. PLoS ONE, 10, e0122297. http://dx.doi.org/10.1371/journal.pone.0122297

[18] Fernandes, J., Weddle, A., Kinter, C.S., et al. (2015) Lysine Acetylation Activates Mitochondrial Aconitase in the Heart. Biochemistry, 54, 4008-4018. http://dx.doi.org/10.1021/acs.biochem.5b00375

[19] Yang, Y., Cimen, H. and Hanetal, M.J. (2010) NAD-Dependent Deacetylase SIRT3 Regulates Mitochondrial Protein Synthesis by Deacetylation of the Ribosomal Protein MRPL10. Journal of Biological Chemistry, 285, 7417-7429. http://dx.doi.org/10.1074/jbc.M109.053421

[20] Cimen, H., Han, M.J., Yang, Y., et al. (2010) Regulation of Succinate Dehydrogenase Activity by SIRT3 in Mammalian Mitochondria. Biochemistry, 49, 304-311. http://dx.doi.org/10.1021/bi901627u

[21] Wei, L., Zhou, Y., Dai, Q., et al. (2013) Oroxylin A Induces Dissociation of Hexokinase II from the Mitochondria and Inhibits Glycolysis by SIRT3-Mediated Deacetylation of Cyclophilin D in Breast Carcinoma. Cell Death \& Disease, 4, e601.

http://dx.doi.org/10.1038/cddis.2013.131

[22] Lagouge, M., Argmann, C., Gerhart-Hines, Z., et al. (2006) Resveratrol Improves Mitochondrial Function and Protects against Metabolic Disease by Activating SIRT1 and PGC-1 a. Cell, 127, 1109-1122. http://dx.doi.org/10.1016/j.cell.2006.11.013

[23] Hallows, W.C., Lee, S. and Denu, J.M. (2006) Sirtuins Deacetylate and Activate Mammalian Acetyl-CoA Synthetases. Proceedings of the National Academy of Sciences of the United States of America, 103, 10230-10235. http://dx.doi.org/10.1073/pnas.0604392103

[24] Mostoslavsky, R., Chua, K.F., Lombard, D.B., et al. (2006) Genomic Instability and Aging-Like Phenotype in the Absence of Mammlian SIRT6. Cell, 124, 315-329. http://dx.doi.org/10.1016/j.cell.2005.11.044

[25] Huang, W., Sherman, B.T. and Lempicki, R.A. (2009) Systematic and Integrative Analysis of Large Gene Lists Using DAVID Bioinformatics Resources. Nature Protocols, 4, 44-57. http://dx.doi.org/10.1038/nprot.2008.211

[26] Narayan, N., Lee, I.H., Borenstein, R., et al. (2012) The NAD-Dependent Deacetylase SIRT2 Is Required for Programmed Necrosis. Nature, 492, 199-204. http://dx.doi.org/10.1038/nature11700

[27] Krishnan, J., Danzer, C., Simka, T., et al. (2012) Dietary Obesity-Associated Hif1Alpha Activation in Adipocytes Restricts Fatty Acid Oxidation and Energy Expenditure via Suppression of the Sirt2-NAD+ System. Genes \& Development, 26, 259-270. http://dx.doi.org/10.1101/gad.180406.111

[28] Jing, E., Gesta, S. and Kahn, C.R. (2007) SIRT2 Regulates Adipocyte Differentiation through FoxO1 Acetylation/Deacetylation. Cell Metabolism, 6, 105-114. http://dx.doi.org/10.1016/j.cmet.2007.07.003

[29] Wang, F. and Tong, Q. (2009) SIRT2 Suppresses Adipocyte Differentiation by Deacetylating FOXO1 and Enhancing FOXO1's Repressive Interaction with PPARgamma. Molecular Biology of the Cell, 20, 801-808. http://dx.doi.org/10.1091/mbc.E08-06-0647

[30] Qiang, L., Wang, L., Kon, N., et al. (2012) Brown Remodeling of White Adipose Tissue by SirT1-Dependent Deacetylation of Ppary. Cell, 150, 620-632. http://dx.doi.org/10.1016/j.cell.2012.06.027

[31] Jiang, S., Wang, W., Miner, J., et al. (2012) Cross Regulation of Sirtuin 1, AMPK and 
PPAR $\gamma$ in Conjugated Linoleic Acid Treated Adipocytes. PLOS ONE, 7, e48874. http://dx.doi.org/10.1371/journal.pone.0048874

[32] Jin, Q., Zhang, F., Yan, T., et al. (2010) C/EBP $\alpha$ Regulates SIRT1 Espression during Adipogenesis. Cell Research, 20, 470-479. http://dx.doi.org/10.1038/cr.2010.24

[33] Qiao, L. and Shao, J. (2006) SIRT1 Regulates Adiponectin Gene Expression through FoxO1-C/Enhancer-Binding Protein Alpha Transcriptional Complex. Journal of Biological Chemistry, 281, 39915-39924. http://dx.doi.org/10.1074/jbc.M607215200

[34] Khan, S.A., Ali, A., Khan, S.A., et al. (2014) Unraveling the Complex Relationship Triad between Lipids, Obesity, and Inflammation. Mediators of Inflammation, 2014, Article ID: 502749. http://dx.doi.org/10.1155/2014/502749

[35] Iyer, A., Fairlie, D.P., Prins, J.B., et al. (2010) Inflammatory Lipid Mediators in Adipocyte Function and Obesity. Nature Reviews Endocrinology, 6, 71-82.

http://dx.doi.org/10.1038/nrendo.2009.264

[36] Catalioto, R.M., Maggi, C.A. and Giuliani, S. (2009) Chemically Distinct HDAC Inhibitors Prevent Adipose Conversion of Subcutaneous Human White Preadipocytes at an Early Stages of the Differentiation Program. Experimental Cell Research, 315, 3267-3280. http://dx.doi.org/10.1016/j.yexcr.2009.09.012

[37] Haberland, M., Carrer, M., Mokalled, M.H., et al. (2010) Redundant Control of Adipogenesis by Histone Deacetylases 1 and 2. Journal of Biological Chemistry, 285, 14663-14670. http://dx.doi.org/10.1074/jbc.M109.081679

[38] Yoo, E.J., Chung, J.J., Choe, S.S., et al. (2006) Down-Regulation of Histone Deacetylases Stimulates Adipocyte Differentiation. Journal of Biological Chemistry, 281, 6608-6615. http://dx.doi.org/10.1074/jbc.M508982200

[39] Sano, M., Minamino, T., Toko, H., et al. (2007) p53-Induced Inhibition of Hif-1 Causes Cardiac Dysfunction during Pressure Overload. Nature, 446, 444-448. http://dx.doi.org/10.1038/nature05602

[40] Potente, M., Ghaeni, L., Baldessari, D., et al. (2007) SIRT1 Controls Endothelial Angiogenic Functions during Vascular Growth. Genes \& Development, 21, 2644-2658. http://dx.doi.org/10.1101/gad.435107

[41] Heather, L.C., Cole, M.A., Tan, J.J., et al. (2012) Metabolic Adaptation to Chronic Hypoxia in Cardiac Mitochondria. Basic Research in Cardiology, 107, 268. http://dx.doi.org/10.1007/s00395-012-0268-2

[42] Fukuda, S., Kaga, S., Zhan, L., et al. (2006) Resveratrol Ameliorates Myocardial Damage by Inducing Vascular Endothelial Growth Factor-Angiogenesis and Tyrosine Kinase Receptor Flk-1. Cell Biochemistry and Biophysics, 44, 43-49. http://dx.doi.org/10.1385/CBB:44:1:043

[43] Chen, R., Dioum, E.M., Hogg, R.T., et al. (2011) Hypoxia Increases Sirtuin 1 Expression in a Hypoxia-Inducible Factor-Dependent Manner. Journal of Biological Chemistry, 286, 13869-13878. http://dx.doi.org/10.1074/jbc.M110.175414

[44] Benigni, A., Corna, D., Zoja, C., et al. (2009) Disruption of the Ang II Type 1 Receptor Promotes Longevity in Mice. Journal of Clinical Investigation, 119, 524-530. http://dx.doi.org/10.1172/JCI36703

[45] Hsu, C.P., Oka, S., Shao, D., et al. (2009) Nicotinamide Phosphoribosyltransferase Regulates Cell Survival through NAD+ Synthesis in Cardiac Myocytes. Circulation Research, 105, 481-491. http://dx.doi.org/10.1161/CIRCRESAHA.109.203703

[46] Pillai, V.B., Sundaresan, N.R., Kim, G., et al. (2010) Exogenous NAD Blocks Cardiac Hypertrophic Response via Activation of the SIRT3-LKB1-AMP-Activated Kinase Pathway. Journal of Biological Chemistry, 285, 3133-3144. 
http://dx.doi.org/10.1074/jbc.M109.077271

[47] Lombard, D.B., Alt, F.W., Cheng, H.L., et al. (2007) Mammalian Sir2 Homolog SIRG3 Regulates Global Mitochondrial Lysine Acetylation. Molecular and Cellular Biology, 27, 88078814. http://dx.doi.org/10.1128/MCB.01636-07

[48] Li, Y., Wen, H., Xi, Y., et al. (2014) AF9 YEATS Domain Links Histone Acetylation to DOT1L-Mediated H3K79 Methylation. Cell, 159, 558-571. http://dx.doi.org/10.1016/j.cell.2014.09.049

[49] Sun, Y., Jiang, X., Chen, S., et al. (2006) Inhibition of Histone Acetyltransferase Activity by Anacardic Acid Sensitizes Tumor Cells to Ionizing Radiation. FEBS Letters, 580, 4353 4356. http://dx.doi.org/10.1016/j.febslet.2006.06.092

[50] Stimson, L., Rowlands, M.G., Newbatt, Y.M., et al. (2005) Isothiazolones as Inhibitors of PCAF and p300 Histone Acetyltransferase Activity. Molecular Cancer Therapeutics, 4, 1521-1532. http://dx.doi.org/10.1158/1535-7163.MCT-05-0135

[51] Wapenaar, H. and Dekker, F.J. (2016) Histone Acetyltransferases: Challenges in Targeting Bi-Substrate Enzymes. Clinical Epigenetics, 8, 59. http://dx.doi.org/10.1186/s13148-016-0225-2

[52] Filippakopoulos, P., Qi, J., Picaud, S., et al. (2010) Seletive Inhibition of BET Bromodomains. Nature, 468, 1067-1073. http://dx.doi.org/10.1038/nature09504

[53] Bertrand, P. (2010) Inside HDAC with HDAC Inhibitors. European Journal of Medicinal Chemistry, 4, 2095-2116. http://dx.doi.org/10.1016/j.ejmech.2010.02.030

[54] Iyer, A., Fenning, A., Lim, J., et al. (2010) Antifbrotic Activity of an Inhibitor of Histone Deacetylases in DOCA-Salt Hypertensive Rats. British Journal of Clinical Pharmacology, 159, 1408-1417. http://dx.doi.org/10.1111/j.1476-5381.2010.00637.x

[55] Adamopoulou, E. and Naumann, U. (2013) HDAC Inhibitors and Their Potential Applications to Glioblastoma Therapy. Oncoimmunology, 2, Article ID: e25219. http://dx.doi.org/10.4161/onci.25219

[56] Chan, V., Fenning, A., Iyer, A., Hoey, A. and Brown, L. (2011) Resveratrol Improves Cardiovascular Function in DOCA-Salt Hypertensive Rats. Current Pharmaceutical Biotechnology, 12, 429-436. http://dx.doi.org/10.2174/138920111794480552

\section{Scientific Research Publishing}

\section{Submit or recommend next manuscript to SCIRP and we will provide best service for you:}

Accepting pre-submission inquiries through Email, Facebook, LinkedIn, Twitter, etc. A wide selection of journals (inclusive of 9 subjects, more than 200 journals)

Providing 24-hour high-quality service

User-friendly online submission system

Fair and swift peer-review system

Efficient typesetting and proofreading procedure

Display of the result of downloads and visits, as well as the number of cited articles

Maximum dissemination of your research work

Submit your manuscript at: http://papersubmission.scirp.org/

Or contact abb@scirp.org 